\title{
Proceso de Atención de Enfermería como instrumento del cuidado, significado para estudiantes de último curso
}

\author{
Nursing Process as the care instrument, \\ meaning to senior nursing students
}

\section{Processo de Atenção de Enfermagem como instrumento do cuidado, significado para estudantes do último curso}

\author{
K.E. Miranda-Limachi ${ }^{\mathrm{a} 1}$, Y. Rodríguez-Núñez ${ }^{\mathrm{b} 2}$, \\ M. Cajachagua-Castro ${ }^{\mathrm{c} *}$ \\ ORCID: \\ a0000-0002-2376-8958 \\ b0o०o-0002-5326-6545 \\ c00oo-0003-3637-8665 \\ ${ }^{1}$ Escuela de Enfermería, Facultad de Ciencias de la Salud, \\ Universidad Peruana Unión, Lima, Perú \\ ${ }^{2}$ Universidad Privada Leonardo Da Vinci, Trujillo, Perú \\ Recibido: 27 marzo 2019/Aceptado: 05 julio 2019
}

RESUMEN

Introducción: El Proceso de Atención de Enfermería (PAE) como guía para el cuidado, se aplica desde que inicia la formación académica. Comprender el significado que tiene para estudiantes del último curso permite visualizar la importancia y dificultades que se presentan en su aplicación, para proponer estrategias que fortalezcan la calidad educativa.

Objetivo: Comprender el significado del PAE que tienen los estudiantes del último curso de Enfermería.

\footnotetext{
*Autor para correspondencia. Correo electrónico: mayela@upeu.edu.pe https://doi.org/10.22201/eneo.23958421e.2019.4.623

1665-7063/@ 2019 Universidad Nacional Autónoma de México, Escuela Nacional de Enfermería y Obstetricia. Este es un artículo Open Access bajo la licencia CC BY-NC-ND (http://creativecommons.org/licenses/by-nc-nd/4.0/).
} 
Método: Estudio con enfoque cualitativo. Referencial teórico y metodológico fue el Interaccionismo Simbólico y la Teoría Fundamentada en los Datos. Muestra por saturación teórica. Colecta de datos con entrevistas semiestructuradas en 17 estudiantes del $10^{\circ}$ ciclo de una universidad privada de Lima Este. Análisis de datos basado en la codificación de Strauss y Corbin posibilitó la identificación de categorías y subcategorías, y el fenómeno central. Resultados: Emergieron cinco categorías: 1) Cuidado enfermero utilizando el PAE desde el inicio de su formación (Condición causal); 2) Satisfacción o insatisfacción al dar cuidado con el PAE (Contexto); 3) PAE como herramienta de investigación en formación profesional (Condiciones Intervinientes); 4) Dificultades en la aplicación del PAE en el cotidiano de los profesionales (Estrategias de Acción/ Interacción) y 5) Mejora de la práctica al dar cuidado con PAE (Consecuencias).

Conclusiones: El fenómeno central es el PAE, instrumento que guía el cuidado de enfermería para brindar a las personas atención integral y con fundamento en evidencia científica; a partir de la experiencia profesional con una proyección positiva al campo laboral, no exenta de limitaciones en su aplicación en el campo hospitalario.

Palabras clave: Educación en enfermería; aprendizaje; atención de enfermería; proceso de enfermería; estudiantes de enfermería; Perú.

\section{ABSTRACT}

Introduction: The Nursing Process (NP) as the guide to care is encouraged from the beginning of the academic formation; and understanding the meaning which this process has to senior nursing students can yield a more precise visualization of the difficulties regarding its application and, consequently, the possibility to promote strategies aimed at strengthening the related quality of education.

Objective: To understand the meaning which NA has to senior nursing students.

Method: This is a study with a qualitative focus. The theoretical and methodological references were the Symbolic Interactionism and the Data Grounded Theory. The sample was formed until theoretical saturation was reached. Data were collected using semi-structured interviews on 17 nursing students in the 1oth cycle of a private university in Lima Este. Based on the Strauss and Corbin coding, the data analysis yielded the categories, subcategories, and main phenomenon.

Results: Five categories emerged: 1) The care using the NP from the beginning of the formation (Causal condition); Satisfaction or insatisfaction while providing care through the NP (Context); NP as a research tool in the professional formation (Intervening conditions); 4) Difficulties while using the NP in the day-to-day activities of the professionals (Action / Interaction strategies); and 5) Improvements in the practice as the result of using the NP (Consequences).

Conclusions: The central phenomenon is the NP which guides the nursing care in the provision of integral attention based on scientific and wide in scope projection.

Keywords: Education, nursing; learning; nursing care; nursing process; students, nursing; Peru.

RESUMO

Introdução: O Processo de Atenção de Enfermagem (PAE) como guia para o cuidado, aplica-se desde que inicia a formação académica. Compreender o significado que tem para 
estudantes do último curso permite visualizar a importância e dificuldades que se apresentam em sua aplicação, para propor estratégias que fortaleçam a qualidade educativa.

Objetivo: Compreender o significado do PAE que têm os estudantes do último curso de Enfermagem.

Método: Estudo com enfoque qualitativo. O referencial teórico e metodológico foi o Interacionismo Simbólico e a Teoria Fundamentada nos Dados. Amostra obtida por saturação teórica. Coleta de dados com entrevistas semiestruturadas em 17 estudantes do $10^{\circ}$ ciclo de uma universidade privada do leste de Lima. A análise de dados baseada em codificação de Strauss e Corbin, possibilitou a identificação de categorias e subcategorias, e o fenómeno central.

Resultados: Emergiram cinco categorias: 1) Cuidado enfermeiro utilizando o PAE desde o início de sua formação (Condição causal); 2) Satisfação ou insatisfação para dar cuidado com o PAE (Contexto); 3) PAE como ferramenta de pesquisa em formação profissional (Condições Interventoras); 4) Dificuldades na aplicação do PAE no cotidiano dos profissionais (Estratégias de Ação/ Interação) e 5) Melhora da prática para dar cuidado com PAE (Consequências).

Conclusões: O fenómeno central é o PAE, instrumento que guia o cuidado de enfermagem para proporcionar às pessoas atenção integral e com fundamento na evidência científica; a partir da experiência profissional com uma projeção positiva no campo laboral, não isenta de limitações em sua aplicação no campo hospitalar.

Palavras chave: Educação em enfermagem; aprendizagem; atenção de enfermagem; processo de enfermagem; estudantes de enfermagem; Peru.

\section{INTRODUCCIÓN}

Uno de los retos actuales es lograr calidad y excelencia en la formación de los recursos humanos en salud $^{1,2}$, en la que se consideren las transformaciones suscitadas en el ámbito político, social y económico, En este sentido, los docentes gestores han realizado importantes esfuerzos para garantizar formación universitaria de calidad ${ }^{3}$, que incorpore los cambios en la salud de las personas y se impulse un equilibrio en los escenarios docente-asistenciales a fin de propiciar el desarrollo de modos de actuación pertinentes 4 . De igual forma, las nuevas necesidades exigen profesionales que sean creativos y resuelvan problemas cambiantes ${ }^{5}$, por consiguiente, es necesario que desde estudiantes aprendan a analizar y evaluar críticamente las necesidades del paciente ${ }^{6}$.

Con base en lo anterior, Enfermería se caracteriza por proporcionar cuidados basados en conocimientos y técnicas específicas7; que permitieron desarrollar un método de trabajo propio, es decir, el Proceso de Atención de Enfermería (PAE), que es un conjunto de procedimientos lógico, dinámico y sistemático para brindar cuidados sustentados en evidencias científicas ${ }^{8}$.

El profesional de enfermería enfoca su trabajo en el cuidado humano basado en el PAE, por medio del cual diagnostica, planifica, ejecuta y evalúa sus acciones, también considera la integralidad, totalidad, seguridad, así como la continuidad requeridas por el sujeto de cuidado en diferentes momentos y en diferentes escenarios ${ }^{9}$. En la atención se da un proceso interactivo entre el cuidador y ser cuidado; el primero tiene el papel activo pues realiza acciones y comportamientos para cuidar. El segundo, ser cuidado, tiene un rol más pasivo y en función de su situación, puede contribuir y ser responsable del propio cuidado en situaciones de educación para la salud ${ }^{10}$.

El aprendizaje en la práctica clínica está caracterizado por una peculiar complejidad que abarca múltiples aspectos de la realidad, caracterizada a su vez por la integración de la teoría con el 
desarrollo de habilidades ${ }^{1,1,2}$. En este contexto, los estudiantes ante igualdad de circunstancias, tienen diferentes niveles de aprendizaje ${ }^{13}$; así que el docente debe motivar al estudiante para que asuma un rol protagónico de liderazgo, con énfasis en brindar cuidado integral y priorizar la parte humana en la relación de ayuda mutua ${ }^{14,15}$. En ciertas ocasiones el estudiante se muestra inseguro en la aplicación del PAE, entonces el docente es depositario de sus angustias, emociones, dudas, dificultades y limitaciones. Algunos ejemplos de las manifestaciones expresadas por los estudiantes son las siguientes: hacer el PAE es tedioso, me parece complicado, el PAE me estresa y se preguntan ¿haremos esto cuando trabajemos con todos los pacientes? ${ }^{16}$.

Las vivencias que experimentan los estudiantes en su práctica clínica son un componente en su formación humanística, técnica y científica, así como para la internalización de la cultura de enfermería ${ }^{17}$; por ello es necesario que desde estudiantes posean conocimientos para el cuidado a fin de que su respuesta emocional, fisiológica, social y espiritual sea de manera oportuna ${ }^{18}$. En este proceso de cuidar se generan vivencias y es importante que éstas sean descritas ${ }^{19}$.

El plan de estudios de la carrera profesional de Enfermería de la Universidad Peruana Unión, integra en el currículo al PAE como metodología de aprendizaje para el cuidado que brinda Enfermería. Desde el primer año toma como referencia los problemas de salud más comunes del medio, los cuales serán abordados durante la práctica clínica y comunitaria, además de una retroalimentación continua durante toda la carrera en las asignaturas de especialidad.

El propósito de este estudio es comprender el significado que tiene el Proceso de Atención de Enfermería para los estudiantes de último año al brindar cuidados, es por ello que en el presente trabajo se describen expresiones, actitudes, manifestaciones y sentimientos que tienen los estudiantes respecto al significado que tienen del PAE. El modelo teórico que surgió de los datos servirá para fortalecer la enseñanza y aprendizaje del PAE, así como adaptar los planes de estudios a las necesidades formativas a fin de garantizar el éxito de los egresados ante las futuras demandas del contexto clínico-laboral ${ }^{20}$.

La Teoría Fundamentada en los Datos (TFD) es el soporte teórico de este estudio, misma que se extrae de las experiencias vividas por los actores sociales respecto a aspectos significativos, lo que posibilita conectar constructos teóricos y potencializar la expansión del conocimiento en enfermería, al igual que el de otras áreas ${ }^{21}$ donde la emergencia de significados es a partir de los datos ${ }^{22}$. El referencial teórico-metodológico fue el Interaccionismo Simbólico, perspectiva teórica que es uno de los abordajes más adecuados para analizar procesos de socialización ${ }^{23}$. La TFD aplicada en enfermería muestra resultados satisfactorios para construir significados sobre la práctica, pues el cuidar requiere interacción transpersonal entre profesionales, con el paciente y la familia ${ }^{24}$.

\section{MÉTODO}

El enfoque del estudio es cualitativo, basado en la Teoría Fundamentada en los Datos y el Interaccionismo Simbólico. La población estuvo conformada por estudiantes de enfermería del $10^{\circ}$ semestre (internos), de la Escuela de Enfermería de la Universidad Peruana Unión, ubicada en la zona este de Lima Perú. Se elige a estudiantes del último año porque al haber aprobado las asignaturas previas de enfermería, tienen mayores vivencias con la aplicación del PAE que los estudiantes de cursos inferiores. Se trabajó con un muestreo no probabilístico y la muestra estuvo constituida por 17 estudiantes, obtenida por saturación teórica. Las investigadoras eran docentes de los estudiantes participantes del estudio. 
Para recolectar los datos se realizó una entrevista en profundidad semiestructurada conformada por cinco preguntas; inició con la obtención de notas registradas en los memos; se seleccionó a los mejores informantes y desde las primeras tres o cuatro entrevistas se fueron generando los códigos. La recolección de datos se desarrolló a través del método de comparación constante donde las investigadoras recogían, codificaban y analizaban los datos en forma simultánea y sucesiva. Al aplicar el muestreo teórico la recolección fue cíclica, es decir, se recogían los datos, se avanzaba y de ser necesario se regresaba al inicio para luego seguir avanzando. Las entrevistas fueron grabadas y transcritas textualmente. La recogida y el análisis de los datos fueron simultáneos. El proyecto fue aprobado por el Comité de Ética de la Universidad Peruana Unión. Los datos fueron analizados bajo el paradigma de codificación de Strauss y Corbin: codificación abierta, codificación axial y codificación selectiva ${ }^{25}$.

El muestreo en la codificación abierta fue para descubrir y categorizar los fenómenos, estuvo abierto a todos los estudiantes y se dio oportunidad al descubrimiento al aprovechar todos los discursos. Mientras que el muestreo en la codificación axial permitió la selección de informantes apropiados para obtener datos que fortalecían las categorías y subcategorías emergentes; el muestreo en la codificación selectiva priorizó a los informantes que proporcionaban datos con conceptos que fortalecieran el fenómeno central al dar información precisa o hasta que ya no aparecieran nuevos datos significativos.

La codificación abierta se inició desde la transcripción de la primera entrevista, donde fueron identificados incidentes que indicaron el camino a seguir en la recolección de datos. En esta etapa fueron nombrados y categorizados los fenómenos por medio de un examen minucioso de los datos, línea por línea, párrafo por párrafo. Los datos fueron descompuestos en partes discretas y fragmentos; se identificaron incidentes, se examinaron y compararon en busca de similitudes y diferencias para generar códigos, que algunas veces fueron llamados códigos en vivo porque fueron utilizadas las palabras de los entrevistados 25 .

Después de la primera aproximación se intentó definir categorías volviendo nuevamente a los datos para profundizar en los códigos identificados, se realizaron agrupamientos en un orden abstracto basado en la capacidad de explicar lo que estaba sucediendo y emergiendo de los datos; que dio como resultado la reducción de los números de códigos. La construcción de las categorías con sus propiedades y dimensiones fue realizada por medio del análisis a profundidad de códigos encontrados. Este proceso permitió identificar y nombrar categorías dándoles especificidad de acuerdo a sus características particulares. Con la codificación axial se relacionaron grupos de categorías y subcategorías, con apoyo del paradigma de codificación, esto posibilitó la sistematización de los datos en relación con la complejidad de estos, para formular explicaciones más precisas y detalladas del fenómeno de estudio, representadas en la óptica de los participantes. En la codificación selectiva, se realizó la reconstrucción teórica de las relaciones entre las categorías encontradas en torno al fenómeno descubierto en el transcurso del proceso investigativo, con esto se logró identificar el fenómeno que, debido a su centralidad explica y da sentido a los datos y sus relaciones.

En la recolección de datos se utilizaron memos, los cuales fueron el registro de ideas y sirvieron para identificar relaciones entre categorías tal como fueron surgiendo. De la misma manera, la codificación selectiva fue plasmada en un diagrama donde se visualizan las categorías y el fenómeno central.

La comprensión del significado del PAE fue facilitada por la aplicación del modelo de Strauss y Corbin cuyos elementos son: condiciones causales, contexto, condiciones intervinientes, estrategias 
y consecuencias, así como la interpretación a la luz de la Interacción Simbólica. Como producto de la aplicación de este método, se establecen modelos teóricos o reflexiones teóricas bajo el paradigma de codificación ${ }^{25}$. El modelo teórico es el resultado obtenido que explica las interacciones entre los investigadores y los participantes.

\section{RESULTADOS}

Con este estudio fue posible comprender el proceso social en el que los internos (estudiantes), atribuyen significados al Proceso de Atención de Enfermería en el campo práctico al captar la intersubjetividad de sus experiencias y la participación activa en la propuesta de mejoras desde el proceso enseñanza - aprendizaje hasta la aplicación del PAE como instrumento para el cuidado, bajo su experiencia desde el inicio de la carrera. Además, se develaron cinco categorías en consonancia con los conceptos teóricos de la interacción simbólica y la dinámica de la teoría fundamentada. De cada categoría emergieron subcategorías. (Figura 1)

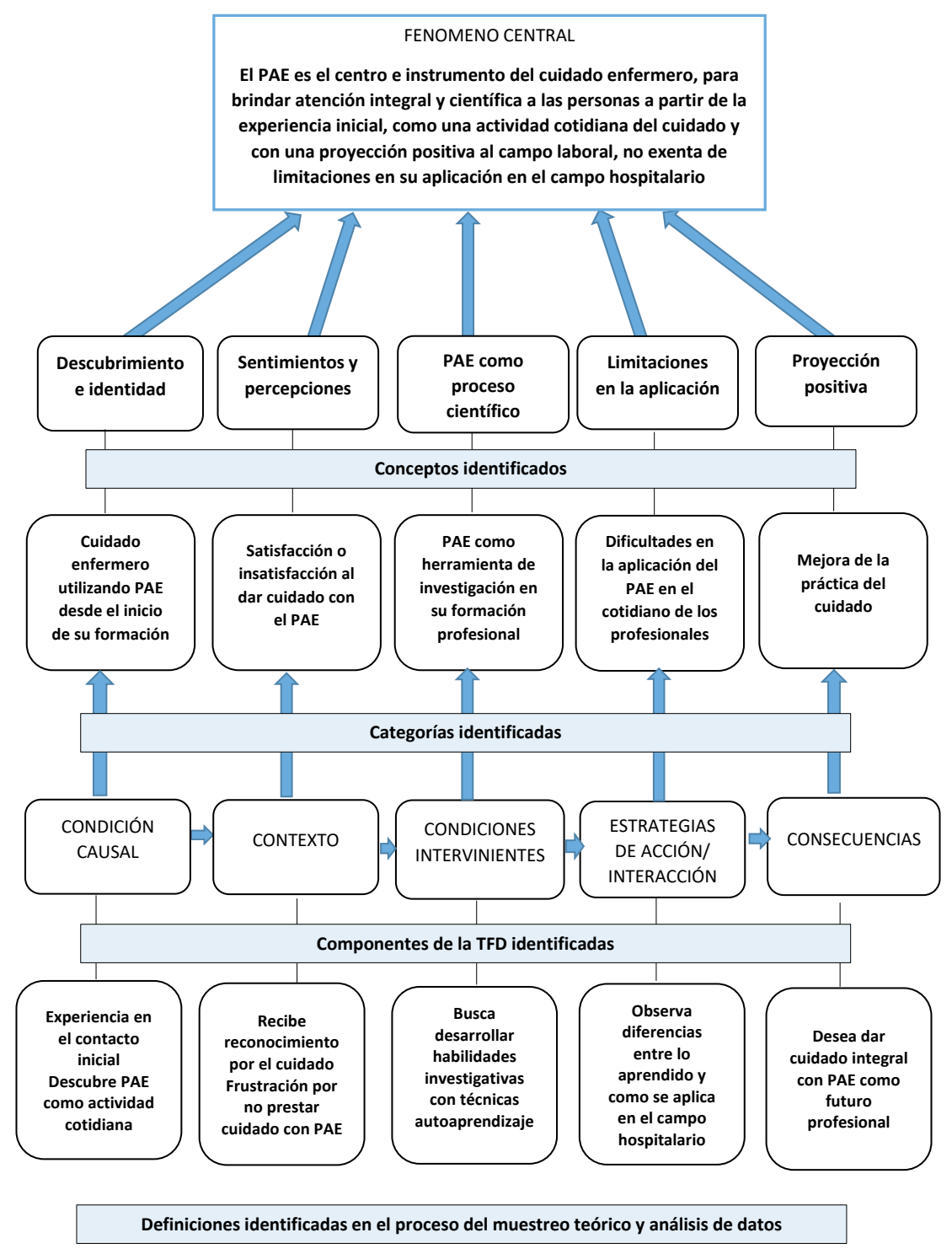

Figura 1. Modelo teórico-explicativo del fenómeno. 
Categoria 1: Cuidado enfermero utilizando el PAE

desde el inicio de su formación académica

El tiempo de estudio de la carrera profesional es un período inicial de transición de un mundo social conocido que debe ser descifrado. Se caracteriza por los inicios del cuidado enfermero a través del aprendizaje continuo con sus propias repercusiones generalizadas. En esta fase el estudiante siente el peso de no estar preparado para responder a las nuevas exigencias en la formación especializada, las cuales requieren un mayor desenvolvimiento, y percibe la discrepancia entre lo que sabe y hace, así como lo que debe saber y hacer, así, el estudiante reconoce que las primeras etapas carecen de familiaridad con el proceso de cuidado de enfermería. La distancia a los esquemas operacionales específicos de la profesión en sí misma puede ser importante para su formación profesional, pues añade nuevas posibilidades a sus conocimientos, construidos a lo largo de su carrera.

Todo lo que el estudiante experimenta desde el inicio de la carrera es vivido de forma intensa y singular, con extremos de sentimientos, valores, actitudes, comportamientos, pensamientos y prácticas, que pueden variar desde el más feliz y tranquilizador hasta los marcados por la ira y la rebelión.

\section{Subcategoría: Contacto inicial con el cuidado.}

Corresponde a la etapa de inserción, al contacto inicial que el estudiante tiene con el cuidado. Muestra cómo los estudiantes inician su formación profesional experimentando diferentes emociones, aprendizajes, situaciones positivas y negativas, con muchas variables que son desconocidas. Se encuentran en un momento en que sus sentimientos impregnan la interpretación de la situación. Frente al inicio del aprendizaje especializado revela que fue acompañado de conflictos internos, muchos de ellos motivados por la información recibida de sus compañeros de años superiores, que en su mayoría eran afirmaciones negativas o infundidas en temor. El contacto inicial con el paciente haciendo uso del PAE revela frustración que es acompañado por una actitud de impulso, rabia, tristeza entre otros sentimientos, y esto es particular a cada experiencia vivida.

Existe un conflicto transferible entre compañeros de estudios; sin embargo, las formas de reacción frente a las vivencias nuevas tienen que ver con las características personales del estudiante y si es capaz de responder, pensando y movilizando una estrategia que busca adaptarse a la situación iniciada hasta finalizarla.

Lo escuché en primer año, los estudiantes de ciclos superiores me dijeron que era lo más fuerte de enfermería, iba a estresarme y cosas así, pero cuando llevé el primer curso de carrera me di cuenta, por lo que nos enseñaron, que es un proceso donde la enfermera o el enfermero tiene que ser muy observador (K1).

\section{Subcategoría: Concepto de PAE como método científico}

Se refiere a que los estudiantes relacionan el PAE como una actividad científica desde el inicio de su licenciatura. Tienen curiosidad por el nuevo aprendizaje adquirido en los inicios de la carrera, para luego sentir que a medida que avanza sus años de estudios, la práctica hace que ese sentimiento de perplejidad se vaya convirtiendo en una actividad metódica y sistemática cotidiana durante su formación profesional.

...Me enseñaron que era un proceso metodológico para el cuidado de enfermería

(K2). 
...Cuando recibí la clase, identifiqué por primera vez que el PAE hace de enfermería una profesión, una ciencia (K8).

\section{Categoría 2: Satisfacción o insatisfacción al dar cuidado con PAE}

El conocimiento y utilización del PAE por los internos de enfermería genera emociones y sentimientos donde los estudiantes experimentan satisfacción o insatisfacción al dar cuidados con el PAE.

\section{Subcategoría: Satisfacción al dar cuidado basado en el PAE}

En este proceso están presentes características, cualidades, situaciones positivas al sentir satisfacción por el cuidado de enfermería brindado con el PAE. Experimentan sentimientos positivos generados por los resultados alcanzados, al recibir reconocimiento del usuario y familia por los cuidados realizados; dado que el cuidado de enfermería es integral, considera el lado humano, la autonomía e individualidad, lo que satisface la necesidad de buen trato, paciencia, comprensión, amor, más allá del propio tratamiento que requiera el paciente.

Interactuaba con él, porque cuanto más interactúas con el paciente le ayudas y satisfaces todas sus necesidades. El cambio emocional ofísico se veía en su recuperación y en el reconocimiento que él nos daba (K4).

Fue muy reconfortante porque estar con la persona a su lado no solamente es confortante para mí, sino para la persona, porque se siente bien que alguien muestre interés por él, en su salud y su bienestar (K1).

La experiencia que tuve fue primero con angustia, un poco de temor quizás, pero luego la experiencia fue agradable para mí, y me llenó de felicidad (K2).

Es en esta interacción que el interno de enfermería identifica que los pacientes y familiares manifiestan su agrado y esto genera sentimientos positivos que identifica como reconocimiento por dar los cuidados que responden a las necesidades específicas, ya que está sirviendo a las personas a través de una abordaje comprensible e individualizado, considerando su parte humana, y no se limita al tratamiento.

\section{Subcategoría: Insatisfacción al dar cuidado con el PAE}

Es generado por el cuidado brindado con el uso del PAE, mismo que representa experiencias y sentimientos negativos. Al desarrollar casos reales en el hospital en sus primeras experiencias de cuidado, los estudiantes manifestaron miedo, temor, estrés, ansiedad y frustración por no tener un amplio conocimiento teórico sobre la salud y enfermedad, lo que dificultaba la aplicación de los cuidados planificados. Estos sentimientos negativos eran internos, propios del estudiante, no por lo que el entorno, los docentes, pacientes o familiares le exigían al respecto.

Sentí algo de estrés, porque tenía que terminar el trabajo de investigación y se juntaron los nervios a la hora de exponer, pero sobre todo, vi que requería mucha responsabilidad con el paciente al momento de ejecutar mis intervenciones, responsabilidad conmigo misma y responsabilidad con mis docentes de práctica (K11).

A mi me frustra que a veces no pueda hacer de todo lo que uno pudiera hacer, porque a veces los cuidados son limitados, sintiendo en ese momento impotencia, frustración por no haber dado el cuidado completo, así como lo aprendido en clase (K12). 
La comprensión de esta subcategoría se torna significativa, porque nos hace reflexionar sobre los aspectos subjetivos, asociados a los sentimientos, ideas y proyectos de vida de cada persona, es decir, las subjetividades individuales. Subjetividad entendida aquí, no psicológicamente, sino en la producción de formas específicas de sociabilidad, de tomar parte de esta construcción y el futuro de los agentes sociales.

\section{Categoría 3: PAE como herramienta de investigación en su formación profesional}

Esta categoría revela el significado que otorgan los internos al PAE como instrumento del cuidado, basado en la mejor evidencia científica en su formación profesional por la práctica del PAE.

\section{Subcategoría: Adquisición de competencia investigativa para el cuidado}

Está relacionado con la experiencia en el desarrollo de trabajos de investigación. En la enseñanza del PAE se realiza investigación formativa durante los diez semestres de estudios, la cual está insertada en las asignaturas disciplinarias donde se fortalece el pensamiento crítico y divergente a través de metodologías participativas, misma que es reconocida por el estudiante como facilitador de aprendizaje que fortalece su competencia en investigación. Este proceso de aprendizaje continuo en su formación académica está marcado por la búsqueda de nuevos conocimientos a través de la investigación.

En algunos casos el aprendizaje fue un tanto memoristico porque teniamos que aprender todos los patrones funcionales, pero lo que más me agradaba era que nos traían casos de estudio y nosotros teníamos que desarrollarlo por grupos, entonces cuando nosotros sustentábamos, otros grupos manejaban mejor el caso, y se discutía y eso hacía que nos fortaleciera y aprendiamos más. También con los ejemplos en el mismo campo de práctica, en el hospital con casos reales (K9).

El PAE significó mucho en mi formación. Yo aprendí porque tuve que investigar diferentes casos, por ejemplo, en el caso del internado, un PAE que me tocó nunca lo había visto en los 4 años, entonces tuve que venir a biblioteca, buscar información sobre la patología, etc.(K5).

\section{Subcategoría: Consolidación de habilidades investigativas con el uso de técnicas}

La carrera de enfermería siendo una disciplina que fundamenta su intervención en el método científico, necesita que desde estudiantes fortalezcan las habilidades de investigación. En este caso se refiere al uso de autoaprendizaje para fijar conocimientos como mecanismo de metacognición. Actualmente las instituciones formadoras enfrentan el desafío de establecer un puente entre la teoría y la práctica, así como fomentar habilidades de pensamiento crítico y reflexivo ${ }^{26}$. En la Escuela de Enfermería desde el primer año se enseña la metodología del PAE, se utilizan técnicas de autoaprendizaje para fijar conocimientos, se aumenta la complejidad con casos de patologías más comunes acordes a la realidad de salud. Estos son resueltos por grupos, y son sustentados generándose un debate; que según los estudiantes, ha sido de mucho beneficio en su aprendizaje, lo que permite consolidar su autoaprendizaje.

[...] Las asignaturas de especialidad, por tener casos clínicos de diversas patologías era una gran ayuda para aprender el PAE, porque en el hospital tú ya sabías qué diagnósticos de enfermería posiblemente podía tener tu paciente. A veces en clases nos parecía como qué, por qué tanta patología, por qué casos clínicos; pero cuando ibas al hospital tenía esa patología tu paciente 
y tú recordabas qué diagnósticos habias elaborado en el salón, qué planes de cuidado habias hecho, y eso te ayudaba a recordar... Entonces yo aprendí en clases con los casos clínicos en grupo; lo haciamos en casa o en la biblioteca, con el NANDA y eso me ayudaba (K10).

\section{Categoría 4: Dificultades en la aplicación del PAE en el cotidiano de los profesionales}

Esta categoría está referida al significado que le dan los estudiantes al PAE al comprobar su utilización por los profesionales de enfermería. Ellos identifican dificultades en la aplicación del PAE en el cuidado que otorgan los enfermeros en el contexto hospitalario donde realizan prácticas.

\section{Subcategoría: Rutina}

Los estudiantes perciben que el cuidado de enfermería brindado por los profesionales en el campo hospitalario difiere de lo aprendido en su formación profesional, ya que observaron que en algunas situaciones no aplican correctamente el PAE. Las actividades son rutinarias y no se aplican todas las fases. Considerando esa realidad, el interno precisa comprender el mundo de la ciencia de enfermería con sus fortalezas y debilidades.

\section{Subcategoría: Factores individuales e institucionales}

Los estudiantes observan que el personal de enfermería no aplica correctamente el PAE en el campo hospitalario. Escuchan a los profesionales de enfermería afirmar que por las muchas actividades administrativas, asistenciales, normas y procedimientos, no aplican correctamente el PAE, y, por lo tanto, encuentran limitaciones individuales e institucionales sobre la aplicación del PAE en el campo profesional. Asimismo, algunos profesionales de enfermería indican a los internos que no hay tiempo para elaborar diagnósticos de enfermería o registrar el cuidado; también observan que dejan de lado el cuidado humanizado, por ejemplo, cuando dan al paciente un saludo protocolar.

En la práctica todo lo aprendido en la teoría, a veces es relacionada, pero en cuanto al PAE, en el hospital ya no siguen los pasos como tú lo aprendiste en el aula (K7).

Las enfermeras cuando valoran al paciente de frente, se van, no le dicen por su nombre, no siempre respetan (K17).

Los internos de enfermería perciben que la utilización del proceso de cuidado de enfermería depende de la estructura organizacional de la institución hospitalaria, además de la decisión personal de la actualización y aplicación del PAE por parte del profesional de enfermería y reconocen que es una dificultad que se debe enfrentar en equipo. A veces el tipo de trabajo de la organización limita la iniciativa al profesional en un desenvolvimiento al proporcionar los cuidados.

Esta categoría es importante, ya que el interno de enfermería en su último año de estudios, observa y experimenta la futura realidad profesional, que en su conocimiento, es diferente muchas veces a lo que aprendió en clases, experiencia de la cual desarrollará estrategias personales para dar cuidado con el PAE cuando sea profesional.

\section{Categoría 5: Mejora de la práctica del cuidado}

El estudiante de último curso considera que el aprendizaje y utilización del PAE, tanto en su vida estudiantil como profesional, permitirá otorgar un cuidado enfermero de calidad. Esta categoría se refiere al significado que le da al PAE al convivir con la realidad en el campo profesional en busca de mejorar su práctica en el cuidado. 


\section{Subcategoría: Cuidado enfermero integral con PAE}

Muestra la relación del estudiante con la realidad del campo profesional y el contexto que lo rodea. En la práctica cotidiana el estudiante reconoce que el PAE es una guía, un método que transciende para orientar sus actividades experimentando sus beneficios para cuidar al ser humano, donde fortalecen el aspecto actitudinal con principios y valores para brindar un cuidado de calidad y con calidez.

Me ayuda a, como enfermera que pueda ser más competitiva, pueda mejorar mis habilidades..., pueda ser una enfermera con amplios conocimientos y sobre todo, diferente a las demás (K3).

Los estudiantes experimentan el beneficio del cuidado integral, deseando mejorar la práctica en el cuidado enfermero a través de la continuidad en los estudios, por eso en su práctica cotidiana, el estudiante reconoce que el PAE es una guía para orientar su actividad: En la satisfacción de las necesidades bio-psico-socio-espirituales de la persona, se considera el entorno familiar, la interacción constante y el trato humano en cada intervención, viendo al ser humano más allá de su propia enfermedad.

El proceso de atención de enfermería nos permite dar un cuidado integral, no tendría sentido si no tuviera el método, es nuestra herramienta principal, que se puede ir perfeccionando con la continuidad de la investigación y mi continuidad en los estudios de posgrado (K2).

El PAE me ayuda a valorar a la persona, no solo verlo como un ser que tiene alguna patología o enfermedad sino verlo de otro punto de vista (K16).

\section{Subcategoría: Proyección positiva como profesional}

Los estudiantes atribuyen un significado al PAE proyectándose para un futuro en el campo profesional, en distintos conceptos, ideas y sentimientos, en el que identifican que es una actividad que realiza en su trabajo cotidiano como estudiante y como futuro profesional. Visualizan el PAE como el corazón de enfermería, ayuda idónea, herramienta principal; es decir, un instrumento que ayuda a cuidar al ser humano de manera integral reconociéndolo como elemento fundamental y facilitador de su trabajo.

Sus acciones de cuidado evidencian conocimientos, habilidades, actitudes de amor, servicio, empatía y trato con dignidad al ser humano, con ética en la interacción con otros, lo que amplía la perspectiva profesional a futuro de los estudiantes.

El proceso de atención de enfermería significa primero el medio de la comunicación y el afecto de paciente - enfermero (K10).

El PAE es un instrumento que se debe utilizar para hacer la valoración correcta al paciente y conocerlo en sí. No es un manual, es una ayuda idónea que tiene que ir contigo en todo momento para brindar un cuidado integral (K4).

Para mi, el PAE, es el corazón del área de enfermería. Enfermería no puede funcionar sin el proceso de atención de enfermería, porque de nada serviría si solo hiciéramos las cosas si nos las dan en una lista de indicaciones (K1).

\section{Descubriendo el fenómeno central}

De los discursos analizados se encuentra que las definiciones identificadas en el proceso del muestreo teórico y análisis de los datos, indican que los sentimientos en el contacto inicial con el cuidado y el descubrimiento del PAE como actividad cotidiana, da lugar al componente Condición causal. El recibir reconocimiento por el cuidado, así como la frustración por no prestar cuidado con PAE permite 
develar el componente Contexto. La búsqueda del desarrollo de sus habilidades investigativas con técnicas autoaprendizaje a través del análisis científico en casos prácticos da lugar al componente de Condiciones intervinientes. La observación de dificultades y diferencias entre lo aprendido y como se aplica el PAE en el campo hospitalario, da lugar al componente Estrategias de Acción-Interacción. La experiencia de conceptualizar positivamente al PAE en su campo laboral proyectándose como futuro profesional preparándose para dar cuidado integral con PAE da lugar al componente Consecuencias.

En este estudio emerge el modelo que propone una conexión e interconexión entre las categorías anteriormente descritas basadas en el modelo de Strauss y Corbin: Cuidado enfermero utilizando el PAE desde el inicio de su formación (Condiciones Causales); Satisfacción personal al dar cuidado con el PAE (Contexto); Limitación para ser aplicado el PAE por profesionales (Condiciones Intervinientes); PAE como herramienta de investigación para el cuidado (Estrategias de Acción/ Interacción) y Mejora de la práctica del cuidado (Consecuencias). Dichas categorías develan los siguientes conceptos: Descubrimiento e identidad, sentimientos y percepciones, fortalecimiento de habilidades investigativas, limitaciones en la aplicación y proyección positiva.

El modelo teórico identificado en este estudio atribuye a que El PAE es el centro e instrumento del cuidado enfermero, para brindar atención integral y cientifica a las personas a partir de la experiencia inicial, como una actividad cotidiana del cuidado y con una proyección positiva al campo laboral, no exenta de limitaciones en su aplicación en el campo hospitalario.

\section{DISCUSIÓN}

El estudio explica los significados atribuidos por los internos de enfermería en su trabajo cotidiano en la última etapa de formación profesional en relación al proceso de atención de enfermería, y cómo ésta orienta sus acciones de cuidado y su proyección al campo laboral como futuros profesionales. Así los internos de enfermería, pasaron por una primera etapa al iniciar la carrera, donde se vieron inmersos en una enseñanza especializada a diferencia de lo vivido anteriormente en la parte académica, iniciando con los Fundamentos de enfermería, donde destaca el método científico: Proceso de Cuidado de Enfermería. Indicando que la relación estudiante-docente, relación entre estudiantes son los núcleos de partida para el devenir de la práctica y utilización del PCE, y es en el mundo hospitalario donde el estudiante pone en práctica lo aprendido en clase.

El significado que tienen los estudiantes sobre el PAE es distinto en diferentes ámbitos. Así Aliafsari et al. sobre análisis de experiencias de estudiantes, identificaron seis categorías: confusión educativa, ausencia de procedimientos de evaluación, oportunidades educativas limitadas, interacciones inapropiadas con el personal de enfermería , cultura de acoso y discriminación ${ }^{27}$. En el presente trabajo no se evidencia confusión educativa sino asombro, perplejidad y encanto con lo nuevo, lo que genera el significado sobre PAE.

Como se mencionó anteriormente, los estudiantes se vieron inmersos en 2 etapas a lo largo de la carrera, mientras que una se caracteriza por la enseñanza especializada, la otra es meramente académica. Con todo, se hizo presente que la relación entre estudiante-docente y la relación entre estudiantes, es el núcleo de partida para la práctica y utilización del PAE. De la misma manera, Lian, Skytt y Engström encontraron que los estudiantes describieron el servicio como un cuidado empoderador que marca la diferencia, pero no sin desafios en diferentes niveles ${ }^{28}$.

Los estudiantes manifestaron que sienten satisfacción por el cuidado proporcionado a través de la aplicación del PAE porque reciben reconocimiento del usuario y familia; comprenden la importancia como cuidadores de salud, cuando su trabajo es reconocido por los usuarios, miembros de equipo, 
docentes y sociedad. Cuando no ocurre este reconocimiento, se genera sufrimiento. Albloushi et al. identificaron que los estudiantes en su experiencia educativa, indicaron que la participación en la atención al paciente, un ambiente acogedor, las habilidades del idioma inglés, la nacionalidad del personal de enfermería y la aceptación por parte del personal de enfermería, los pacientes y otros profesionales de la salud, son algunos de los factores que afectan el sentido de los estudiantes ${ }^{29}$. Así mismo Lian, Skytt y Engström encontraron que, para los estudiantes, el servicio tuvo un impacto en ellos; el apoyo interno y / o externo fue estimulante y gratificante, mientras que el apoyo deficiente fue frustrante y los hizo sentir impotentes. Cabe señalar que los autores enfatizaron la necesidad de mejoras a diferentes niveles ${ }^{28}$.

Se encontró que algunos entrevistados se sintieron insatisfechos con el cuidado brindado al aplicar el PAE por diversos factores, como tiempo, ausencia de acompañamiento durante el aprendizaje en el campo práctico o porque no recibieron reconocimiento por los esfuerzos realizados. En un estudio realizado por Ahn y Choi encontraron que existe falta de modelos a seguir ${ }^{30}$. Ahora bien, detectamos una situación parecida en el grupo de estudio, ya que en la práctica hospitalaria los profesionales de enfermería no otorgan cuidado con PAE, de esta manera no son modelos a seguir, lo que genera incertidumbre en los estudiantes e insatisfacción.

La carrera de Enfermería está basada en sólidos conocimientos científicos y los estudiantes adquieren dicho concepto del PAE como método científico, lo que les trae solidez al dar cuidado. Con respecto a las experiencias vividas por los estudiantes, Aleshire, Dampier y Woltemberg, identificaron que los docentes deben crear y evaluar intervenciones innovadoras de enseñanza para mejorar la preparación de los estudiantes para ser miembros efectivos del equipo ${ }^{31}$. El aprendizaje del PAE se torna fundamental para los estudiantes para fomentar el pensamiento reflexivo, razonamiento y juicio clínico. A su vez, la búsqueda de información con criterio estimula la comprensión y va construyendo significados durante su formación. Esto ayuda a desarrollar su competencia investigativa y entender el porqué de sus acciones.

El estudiante adquiere experiencia al aplicar el PAE en un contexto real, lo que conlleva a proyectarse al futuro, para seguir preparándose, y seguir esforzándose para brindar cuidados integrales. En ese sentido, el PAE como instrumento trae beneficios para el cuidado del ser humano y para sí mismo porque posibilita una atención integral a los pacientes, no solo atendiendo las necesidades físicas sino también las emocionales, espirituales y sociales. De la misma manera posibilita la interacción con la familia del usuario, mediante un trabajo coordinando con el equipo de salud, definiendo acciones/interacciones, que puedan realizar un cuidado organizado, buscando simplificar y garantizar cualidades del enfermero.

El mundo hospitalario es donde el estudiante pone en práctica lo aprendido, es un terreno desconocido e incierto. Las vivencias que allí experimenta son el pilar para su formación integral y para la internalización de la cultura enfermera. El inicio de la práctica clínica se presenta como un momento incierto para el estudiante, experimenta vivencias con inseguridad, temor y miedo a lo desconocido. El primer contacto con la realidad hospitalaria o comunitaria es vital para apaciguar dichos temores y sentir alivio; una de las principales preocupaciones en aquel momento de la experiencia práctica es conocer a la enfermera/tutor/a de la práctica clínica o comunitaria.

A medida que el estudiante va cursando la carrera se va relacionando con el PAE. El proceso de aprendizaje inicial en su práctica soporta una doble carga, deben aprender tanto a ejecutar interpretaciones del cuidado de enfermería, como a reconocer su ejecución competente; estos dos componentes de la tarea de aprendizaje se apoyan mutuamente. Los estudiantes también reconocen 
que es fundamental aplicar el PAE para garantizar un cuidado de calidad, ya que es un instrumento importante que le da fundamento a su práctica y apoya el quehacer profesional de enfermería basado en la continuidad ayudando a unificar criterios profesionales y es, además, un instrumento adecuado para la investigación.

El estudio explica los significados atribuidos por los estudiantes en su trabajo cotidiano en la última etapa de formación profesional en relación al PAE, y cómo ésta orienta sus acciones de cuidado y su proyección al campo laboral. De igual forma, Murrain encontró que los estudiantes identifican los atributos que debe tener todo profesional de enfermería ${ }^{32}$ para fortalecer su formación profesional.

Los hallazgos evidencian la necesidad de identificar los puntos críticos en las prácticas hospitalarias a fin de que los profesionales de enfermería asuman su rol de modelo en la atención del paciente con el PAE. A su vez el estudio permite plantear el fortalecimiento de una cultura de calidad en el desarrollo de sus funciones como estudiantes de último curso, superando las vivencias en las prácticas respecto a la aplicación del PAE por los profesionales. Asimismo, se recomienda ampliar el estudio al área comunitaria a fin de identificar el significado del PAE en ese ámbito, detectar limitaciones y así promover altos estándares en la calidad educativa de enfermería. Gracias a que para los estudiantes de último curso consideran que aplicar el PAE es dar cuidado enfermero basado en conocimientos científicos, se propone fortalecerlo con el enfoque de Enfermería Basada en la Evidencia.

Con el propósito de fortalecer el significado del PAE en el cuidado enfermero para atender las respuestas humanas, se propone la utilización del PAE informatizado o el uso de aplicativos, lo que disminuiría las desventajas al dar atención con el PAE cuando los estudiantes de último curso sean profesionales, ya que su utilización desde la época de formación promueve la autonomía del profesional de enfermería.

\section{CONCLUSIONES}

El fenómeno central identificado es: el PAE centro e instrumento del cuidado enfermero, para brindar atención integral y científica a las personas a partir de la experiencia inicial, como una actividad cotidiana del cuidado y con una proyección positiva al campo laboral, no exenta de limitaciones en su aplicación en el campo hospitalario. El significado del PAE se presenta a nivel simbólico de interacción considerándolo como el componente central del cuidado, el instrumento o herramienta principal para brindar salud a las personas, utilizado cotidianamente en el campo clínico, integrando la conceptualización del mismo en el presente y proyectando su aplicación en su labor como futuro profesional.

El PAE se desvela como un fenómeno en movimiento, cuando los estudiantes entran en contradicción con lo aprendido al observar que los profesionales de enfermería que trabajan en los hospitales no aplican de manera correcta el PAE, lo que ocasiona insatisfacción.

\section{RESPONSABILIDADES ÉTICAS}

Protección de personas y animales. Los autores declaran que para esta investigación no se han realizado experimentos en seres humanos ni en animales.

Confidencialidad de los datos. En este artículo no aparecen datos confidenciales de los participantes. Derecho a la privacidad y consentimiento informado. El trabajo se realizó con la aprobación del Comité de Ética de la Universidad Peruana Unión y se obtuvo el consentimiento informado de cada uno de los participantes del estudio.

Financiamiento. No se recibió ningún tipo de financiamiento para la presente investigación. Conflictos de intereses. Las autoras informan que no existen conflictos de interes. 


\section{REFERENCIAS}

1. Gómez-Aguado R, Díaz-Díaz BY, Fernández-Camargo I, Naithe-Pérez D. Percepción de estudiantes sobre el proceso enseñanza aprendizaje en la asignatura de Enfermería Pediátrica. Rev haban cienc méd. 2016; 15(4): 630-41. http://bit.ly/34anvfq

2. Roque-Herrera Y, Gafas-González C, Herrera-Molina AS, Salazar-Granizo Y, Betancourt-Jimbo CR, Figueredo-Villa K . Pertinencia de la formación académica de enfermería. Universidad Nacional de Chimborazo. Ecuador. Educ. med. 2018; 19(S2): 73-8. https://doi.org/10.1016/j.edumed.2017.04.010

3. Da Cunha-Santos K, De Andrade SR, Erdmann-Lorenzini A. Enfermero gestor universitario: una teoría fundamentada en los datos. Rev. Lat.-Am. Enferm. 2018; 26(0):e2980.

http://dx.doi.org/10.1590/1518-8345.2199.2980

4. Pernas-Gómez M, Zubizarreta-Estévez M, Leyva-Hidalgo M. Escenarios docentes asistenciales para la formación de los enfermeros en Cuba. Educ Med Super 2010; 24(4): 504-15. http://bit.ly/2q2zJHS

5. González-Hernando C, Martín-Villamor PG, Martín-Durántez N, López-Portero S. Evaluación por los estudiantes al tutor de enfermería en el contexto del aprendizaje basado en problemas. Enferm. univ.. 2015; 12(3): 110-5. https://doi.org/10.1016/j.reu.2015.07.002

6. Kuiper R, Pesut D, Kautz D. Promoting the self-regulation of clinical reasoning skills in nursing students. Open Nurs J 2009; 3: 76-85. http://dx.doi.org/10.2174/1874434600903010076

7. Ledesma-Delgado ME. Significado atribuído ao processo de enfermagem por enfermeiras de um hospital no México: entre o fazer rotineiro e o pensar idealizado. [Tese de Doutorado]. Ribeirão Preto, Brasil: Escola de Enfermagem de Ribeirão Preto, Universidade de São Paulo; 2008. https://doi.org/10.11606/T.22.2008.tde-25032008-164942

8. Jara-Sanabria F, Lizano-Pérez A. Aplicación del proceso de atención de enfermería por estudiantes, un estudio desde la experiencia vivida. Enferm. univ. 2016; 13(4): 208-15.

https://doi.org/10.1016/j.reu.2016.08.003

9. Carvajal-Hermida EY, Sánchez-Herrera B. Nursing care with a human approach: a model for practice with service excellence. Aquichan. 2018; 18(2): 149-59. http://bit.ly/2q2EdOJ

10. Waldow VR. Cuidar: expresión humanizadora de la enfermería. México, D.F.: Palabra Ediciones; 2008.

11. Valadares-Valente G, Viana-De Oliveira L. O processo de formação na especialidade a partir da experiência do enfrentamento do conhecimento novo. Cogitare Enferm. 2009; 14(2): 379-83. http://dx.doi.org/10.5380/ce.v14i2.15668

12. Milton-Wildey K, Kenny P, Parmenter G, Hall J. Educational preparation for clinical nursing: The satisfaction of students and new graduates from two Australian universities. Nurse Educ Today. 2014; 34(4): 648-54. https://doi.org/10.1016/j.nedt.2013.07.004

13. Bernal-Becerril ML, Sánchez-Arce LE. Significado del cuidado obstétrico desde el cotidiano del estudiante de enfermería. Enferm. univ. 2014;11(3): https://doi.org/10.1016/S1665-7063(14)72670-3

14. Faustino-Herculano RL, Egry-Yoshikawa E. A formação da enfermeira na perspectiva da educação - reflexões e desafios para o futuro. Rev. Esc. Enferm. USP 2002; 36(4): 332-7.

https://doi.org/10.1590/S0080-62342002000400006

15. Islas-Salinas P, Pérez-Piñón A, Hernández-Orozco G. Rol de enfermería en educación para la salud de los menonitas desde el interaccionismo simbólico. Enferm. univ. 2015; 12(1): 28-35.

https://doi.org/10.1016/j.reu.2015.05.005

16. Rodríguez-García M, Ruiz-López M, González-Sanz P, Fernández-Trinidad M, De Blas-Gómez I. Experiencias y vivencias del estudiante de $4^{\circ}$ de enfermería en el practicum. Cult. cuid. 2014; 18(38): 25-33. http://dx.doi.org/10.7184/cuid.2014.38.06 
17. Rivera-Alvarez LN. Saber y experiencia de el/la estudiante de enfermería en sus prácticas de cuidado. [Tesis de doctorado] . Barcelona, España: Facultad de Pedagogía, Universitat de Barcelona; 2013 http://bit.ly/2JoFfvx

18. Carrillo-Algarra AJ, García-Serrano L, Cárdenas-Orjuela CM, Díaz-Sánchez IR, Yabrudy-Wilches N. La filosofía de Patricia Benner y la práctica clínica. Enferm. glob. 2013; 12(4): 346-61. http://bit.ly/2pbS2uu

19. Mendes MA., Bastos-Ribeiro MA. Processo de Enfermagem: seqüências no cuidar, fazem a diferença. Rev. Bras. Enferm . 2003; 56(3): 271-6. http://dx.doi.org/10.1590/So034-71672003000300011

20. Rodríguez-García MC. Percepción de los estudiantes del Grado en Enfermería sobre su entorno de prácticas clínicas: un estudio fenomenológico. Enferm. clín. 2018; 29(5):264-70 https://doi.org/10.1016/j.enfcli.2018.10.004

21. De Carvalho C, Leite J, Soares de Lima SB, Conceição MA. Teoría fundamentada en los datos-Aspectos conceptuales y operacionales: un método posible para ser aplicado en la investigación en enfermería. Rev Latino-Am Enfermagem. 2009; 17(4):573-9.http://dx.doi.org/10.1590/So104-11692009000400021

22. Cuñat R. Aplicación de la teoría fundamentada (Grounded Theory) al estudio del proceso de creación de empresas. XX Congreso anual de AEDEM, Vol. 2, 2007. https://bit.ly/2BMb7pJ

23. De Carvalho VD, De Olivera L P do RD. Interacionismo simbólico: origens, pressupostos e contribuições aos estudos em Psicologia Social. Psicol Cienc Prof 2010; 30(1):146-61. https://doi.org/10.1590/S1414-98932010000100011

24. Aires de Freitas-Lopes $\mathrm{CH}$, Bessa-Jorge MS. Interaccionismo simbólico e a posibilidade para o cuidar interativo em enfermagem. Rev Esc Enferm. U S P 2005; 39(1):103-8. https://doi.org/10.1590/Soo80-62342005000100014

25. Strauss A, Corbin J. Bases de la investigación cualitativa: técnicas y procedimientos para desarrollar la teoría fundamentada. Medellín, Colombia: Ed Universidad de Antioquía; 2002. 341 https://bit.ly/2qKx5Hc

26. Jara V, Castro J. Desarrollo de juicio clínico con mapas conceptuales de cuidado: experiencia de estudiantes de enfermería. Enferm. univ. 2017;14(4):259-65. http://dx.doi.org/10.1016/j.reu.2017.09.003

27. Aliafsari-Mamaghani E, Rahmani A, Hassankhani H, Zamanzadeh V, Campbell S, Fast O, et al. Experiences of Iranian nursing students regarding their clinical learning environment. Asian Nurs Res (Korean Soc Nurs Sci). 2018;12(3):216-22. https://doi.org/10.1016/j.anr.2018.08.005

28. Lian Z, Skytt B, Li C, Engström M. Nursing students' reflections on caring for end-of-life patients in a youth volunteer association. Nurse Educ Pract. 2019;34:204-9. https://doi.org/10.1016/j.nepr.2018.12.006

29. Albloushi M, Ferguson L, Stamler L, Bassendowski S, Hellsten L, Kent-Wilkinson A. Saudi female nursing students experiences of sense of belonging in the clinical settings: A qualitative study. Nurse Educ Pract. 2019; 35:69-74. https://doi.org/10.1016/j.nepr.2019.01.008

30. Ahn YH, Choi J. Incivility experiences in clinical practicum education among nursing students. Nurse Educ Today. 2019; 73:48-53. https://doi.org/10.1016/j.nedt.2018.11.015

31. Aleshire ME, Dampier A, Woltenberg L. Evaluating undergraduate nursing students' attitudes toward health care teams in the context of an interprofessionally-focused nursing course. J Prof Nurs. 2018;35(1):37-43. https://doi.org/10.1016/j.profnurs.2018.05.004

32. Murrain-Knudson E. Visibilidad y calidad del cuidado ¿consecuencia del nivel de profesionalismo? Repert Med y Cirugía. 2017; 26(4): 231-41. https://doi.org/10.1016/j.reper.2017.10.003 